\title{
El uso de experimentos como método de aprendizaje activo en cursos de iniciación a la Economía: una experiencia*
}

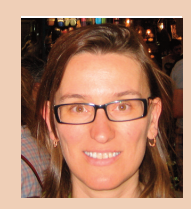

Yadira González de Lara

Profesora Ayudante Doctor en la Universidad de Valencia e investigadora dentro del proyecto de excelencia PROMETEO en la Universidad CEU-Cardenal Herrera

yadira.gonzalez@uv.es

|Fecha presentación: 03/02/2012 | Aceptación: 26/04/2012 |Publicación: 20/06/2012

\begin{abstract}
Resumen
Este trabajo presenta una experiencia docente del uso de experimentos como método de aprendizaje activo y facilita su implementación al ofrecer materiales de apoyo al libro de Bergstrom y Miller (2008). Partiendo del experimento sobre un mercado perfectamente competitivo, se han elaborado (i) una presentación en Power Point con las instrucciones, (ii) un programa en Excel que confiere al profesor pleno control sobre la actividad y permite realizar el experimento, presentar los resultados y explicar la teoría correspondiente en una única clase y (iii) una serie de ejercicios evaluables para que los alumnos afiancen en casa las competencias y habilidades aprendidas experimentando.
\end{abstract}

Palabras clave: aprendizaje activo, economía experimental, mercados perfectamente competitivos

\section{Resum}

Aquest treball presenta una experiència docent de l'ús d'experiments com a mètode d'aprenentatge actiu i facilita la seva implementació en oferir materials de suport al llibre de Bergstrom i Miller (2008). Partint de l'experiment sobre un mercat perfectament competitiu, s'han elaborat (i) una presentació en Power Point amb les instruccions, (ii) un programa en Excel que confereix al professor ple control sobre l'activitat i permet realitzar l'experiment, presentar els resultats i explicar la teoria corresponent en una única classe i (iii) una sèrie d'exercicis avaluables perquè els alumnes afermin a casa les competències i habilitats apreses experimentant.

Paraules clau: aprenentatge actiu, economia experimental, comprats perfectament competitius

\section{Abstract}

This work introduces Economics experiments in the classroom as a means to achieve active learning and facilitates the implementation of the experiments proposed by Bergstrom and Miller (2008) by providing complementary teaching materials. In particular, it provides (i) a power point presentation of their market experiment under perfect competition, (ii) an Excel file that endows the instructor with full control over the activity and enables him/her to conduct the experiment, present the results and place them within the theory in one class of about 100 minutes, and (iii) various exercises to be done by the students as homework and graded by the instructor.

Keywords: Active Learning, Experimental Economics, Markets under Perfect Competition

* Una versión preliminar de este trabajo fue presentada en las II Jornadas de Docencia en Economía celebradas en Valencia en abril de 2010. Quiero agradecer los comentarios de Juan Vicente Llinares, César Nebot y Héctor Solaz, la participación de Penélope Hernández y Maite Pastor en la ejecución de los primeros experimentos y la cita de Confucio que Jesús Sánchez quiso compartir conmigo. Parte de este trabajo fue financiado por la Generalitat Valenciana con cargo al proyecto GV-PROMETEO/2008/106 y por la Universidad CEU-Cardenal Herrera con cargo al centro de Elche. 


\section{Introducción}

Durante mucho tiempo hemos enseñado Economía haciendo uso casi exclusivo de herramientas teóricas y clases magistrales. En gran medida ello se ha debido a la opinión generalizada de que la Economía, al igual que otras Ciencias Sociales, es antitética a la experimentación y al uso de laboratorios. Esta opinión, sin embargo, está siendo cuestionada tanto desde el punto de vista científico como docente. Por una parte, la concesión del Premio Nobel en el 2002 a Vernon Smith por "haber establecido los experimentos de laboratorio como una herramienta en el análisis económico empírico" ha conferido plena legitimidad a la Economía Experimental como área de investigación ${ }^{1}$. Por otra parte, un sin fin de estudios pedagógicos han demostrado que el aprendizaje activo mejora los rendimientos y la actitud de los estudiantes, lo que aconseja la adopción de estrategias alternativas que involucren más activamente a los estudiantes en el proceso de aprendizaje (véanse las referencias bibliográficas en Durham et al. 2007)

Por todo ello, cada vez con mayor asiduidad se proponen experimentos de Economía en clase. La oportunidad de participar en un entorno de mercado controlado y de observar las fuerzas del mercado ofrece al estudiante una experiencia de aprendizaje muy distinta a la de las clases magistrales en las que el profesor se limita a explicar cómo actúan esas fuerzas (Bergstrom y Miller 2008: iv). Al comparar ambas estrategias docentes, varios trabajos de investigación han hallado un efecto positivo del aprendizaje experimental sobre la adquisición y retención del conocimiento por parte de los alumnos así como sobre su actitud y satisfacción. Los alumnos que experimentaron, en media, obtuvieron mejores puntuaciones en los exámenes y valoraron más favorablemente a sus profesores que los grupos de control en los que no se realizaron experimentos (Emerson y Taylor 2004; Ball et al. 2006; Dickie 2006; Durham et al. 2007). Sin embargo, no debe confundirse la incorporación del aprendizaje experimental a nuestro kit de herramientas con la eliminación de las clases magistrales. En todos los casos analizados las sesiones con experimentos incluyeron una fase de discusión en la que el profesor situó los resultados experimentales en su contexto teórico. Tal y como sugiere la Teoría del Conocimiento, el uso combinado de experimentos y lecciones mejora el aprendizaje puesto que permite presentar un mismo concepto desde distintos ángulos y repetir las mismas ideas en varios contextos (Emerson y Taylor 2004: 683). Asimismo, permite satisfacer las necesidades cognitivas de todos los alumnos, independientemente de cuál sea su estilo de aprendizaje preferido (Durham et al. 2007: 165).

Un gran número de los experimentos que se proponen en el aula se basan en el excelente manual de Bergstrom y Miller (2008), cuya primera edición en inglés se publicó en 1997. Estos autores ofrecen la posibilidad de organizar un curso completo sobre Experimentos con los principios económicos o de incorporar alguno de los experimentos propuestos como suplemento a un curso más tradicional de Microeconomía. Los materiales que ofrecen facilitan el uso combinado de experimentos, lecciones teóricas y lecciones prácticas por parte de profesores que aprecian los logros académicos que brinda esta estrategia docente aún sin ser experimentalistas ellos mismos ni disponer de un laboratorio. No obstante, la ejecución de dichos experimentos, tal y como se proponen en el manual de Bergstrom y Miller (2008), resulta compleja para algunos profesores y requiere que los alumnos dediquen una buena parte de las horas de trabajo presencial al aprendizaje experimental. El alto coste de iniciación para los profesores y el elevado coste de oportunidad para los alumnos de realizar estos experimentos han ido en detrimento de su uso, lo que ha privado a docentes y estudiantes de sus muchos beneficios pedagógicos.

Este trabajo ofrece una serie de materiales de apoyo al capítulo 1 del manual de Bergstrom y Miller (2008) que reducen dichos costes e incentivan así la incorporación del aprendizaje experimental a las estrategias docentes de cursos de iniciación a la Economía ${ }^{2}$. La eficacia y eficiencia de estos materiales ha sido probada en la Universidad CEUCardenal Herrera y en la Universidad de Valencia, en las que varios profesores que nunca habían realizado experimentos en clase se atrevieron a hacerlo, siendo capaces de conducir el experimento, discutir los resultados, presentar el modelo básico sobre competencia perfecta, confrontar la evidencia experimental con las predicciones teóricas y distribuir una serie de ejercicios para que los alumnos afiancen en casa los conocimiento adquiridos en clase en una única sesión de 100 minutos. Al reducir los costes de implementación y aprovechar el entusiasmo que los alumnos experimentan para captar su atención durante la fase explicativa de la actividad docente, estos materiales aumentan la rentabilidad del uso docente de los experimentos.

\section{Objetivos}

Tomando como punto de partida el capítulo 1 del libro de Bergstrom y Miller (2008) sobre Experimentos con los principios económicos y el manual para el profesor disponibles en el área de personal docente de la página Web de la editorial (http://www.antonibosch.com), se ha diseñado, ejecutado y coordinado una actividad docente cuyos objetivos generales y específicos se enumeran en la Tabla 1.

\section{El diseño de la actividad docente}

Para alcanzar dichos objetivos, la experiencia docente realizada combinó los experimentos con lecciones magistrales y clases prácticas puesto que, como la literatura académica sobre el uso docente de experimentos demuestra (e.g. Ball et al. 2006, Dickie 2006; Durham et al. 2007; Emerson y Taylor 2004), la mejora de los rendimientos y de la actitud de los alumnos ante el aprendizaje experimental requiere una fase de discusión en la que el profesor sitúe los resultados experimentales en su contexto teórico y una fase de aprendizaje (evaluable) en el que el alumno afiance dichos conocimientos.

Hubo, por tanto, que seleccionar qué experimento implementar y en qué cursos hacerlo. Se seleccionó el experimento sobre la oferta y la demanda propuesto por Bergstrom y Miller (2008; capítulo 1) dado el mayor impacto que ha demostrado tener sobre el aprendizaje en varias universidades americanas (Durham et al. 2007:175) y

\footnotetext{
${ }^{1}$ Para más información, véase la página Web oficial de los Premios Nobel http://nobelprize.org/nobel_prizes/economics/laureates/2002.

2 Llinares Císcar y Nebot Monferrer (2010) aportan unos materiales similares para facilitar la implementación del experimento sobre selección adversa propuesto por Bergstrom y Miller (2008).
} 


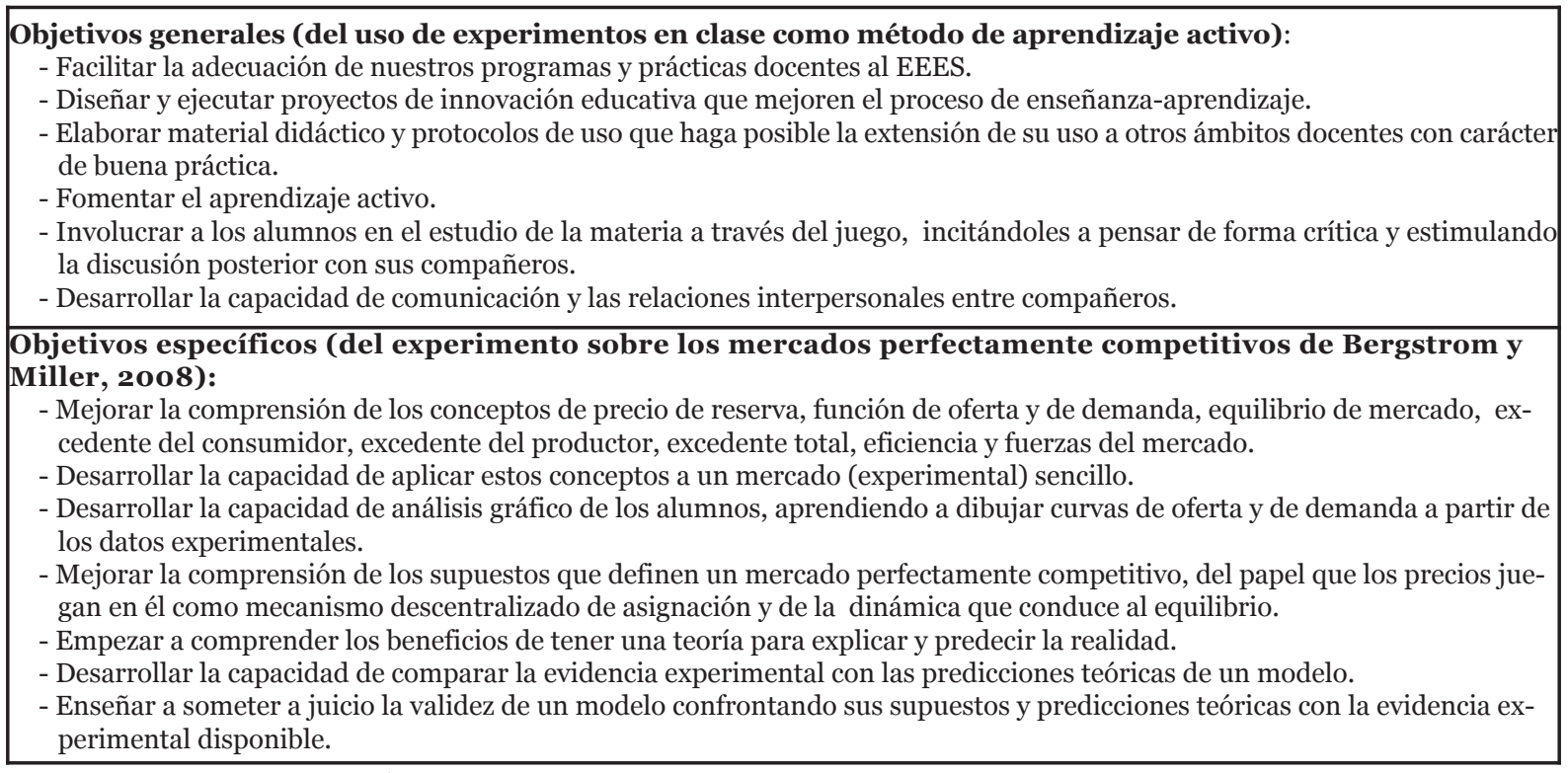

Tabla 1: Objetivos generales y específicos de la actividad docente propuesta.

la posibilidad de implementarlo en todos los cursos de iniciación a la Economía que imparte la Universidad CEU-Cardenal Herrera y que coordina la autora de este trabajo. De esta forma se pudo explotar los beneficios del aprendizaje experimental desde el inicio de cada curso y se permitió la extensión del uso de los experimentos a varias titulaciones, lo que, a la luz de la literatura sobre el tema (e.g. Ball et al. 2006: 445), se espera mejore la actitud de los estudiantes frente a la Economía y consiga así engancharles en el estudio individual de la materia y en la discusión de los experimentos con sus compañeros de clase.

Brevemente, el experimento propuesto consiste en reproducir un mercado perfectamente competitivo en el que cada estudiante pueda, según el papel que se le asigne, comprar o vender una naranja en cada ronda de negociación en la que el mercado esté abierto. El experimento consta de dos sesiones, cada una de tres rondas, en las que los parámetros que definen las curvas de oferta y demanda cambian, reflejando un shock tanto de oferta como de demanda. Los estudiantes pueden así observar y experimentar cómo se comporta el mercado y confrontar, con la ayuda del profesor, la evidencia experimental con las predicciones de la teoría económica.

Asimismo, fue necesario elaborar una serie de materiales didácticos que facilitaran la implementación de la actividad, permitieran la extensión de su uso a otros docentes y redujeran el tiempo de ejecución del experimento. Estos materiales, que se describen a continuación, están disponibles en la Plataforma de Docencia en Economía (http://www.pdocencia.com). Alternativamente, se pueden obtener solicitándolos directamente a la autora.

- El protocolo de uso (protocolo.doc) describe con todo detalle los materiales y procesos necesarios para implementar la actividad docente, incluyendo la ejecución del experimento, la discusión de los resultados a la luz de la teoría y la posterior resolución de las tareas encomendadas a los alumnos como trabajo para casa.
- Los materiales para los alumnos incluyen las hojas de información personal (HIP.doc) que especifican los parámetros con los que jugarán en el experimento y los ejercicios (ejercicios.pdf) que deberán resolver antes de empezar a jugar (a modo de calentamiento) y después del juego (para afianzar en casa las competencias y habilidades aprendidas experimentando). El profesor deberá calcular el número máximo de alumnos que podrían asistir al experimento y fotocopiar tantos paquetes completos de seis hojas de información personal (HIP) como fueran necesarios (una hoja por alumno). A continuación deberá numerar cada HIP de forma que el tipo $\mathrm{A} / \mathrm{B} / \mathrm{C} / \mathrm{D} / \mathrm{E} / \mathrm{F}$ del primer paquete corresponda al número $1 / 2 / 3 / 4 / 5 / 6$, el del segundo paquete al $7 / 8 / 9 / 10 / 11 / 12$ y así sucesivamente, tal y como se indica en el protocolo de uso. La numeración de las HIP permite identificar a los alumnos de forma anónima, registrar las transacciones realizadas por ellos mucho más rápido y calcular automáticamente los beneficios (excedentes) obtenidos por cada uno de ellos mediante el fichero Excel $<$ exp_naranjas.xls $>$.

- Los materiales para el profesor incluyen una presentación en Power Point (presentación.ppt) donde se explican las reglas del juego y un fichero Excel (exp_naranjas.xls) que confiere al profesor pleno control sobre el experimento y permite realizar la actividad docente en una clase de $100 \mathrm{mi}-$ nutos. Este fichero está compuesto por varias hojas, todas ellas protegidas para evitar cambios indeseados durante la celebración del experimento ${ }^{3}$. En la hoja $<$ Tipos $>$ el profesor deberá anotar en las casillas azules el número de paquetes completos de seis y el tipo (A-F) de la última hoja de información personal que haya distribuido. Automáticamente se generará la Tabla 1 (ver Figura 1) con el número de oferentes y de demandantes de cada tipo ${ }^{4}$.

Al finalizar el experimento, los alumnos deberán copiar estos datos en la hoja de ejercicios correspondiente y dibujar a partir de ellos las curvas de oferta y de demanda escalona-

\footnotetext{
${ }^{3}$ Si quisiera mejorar el fichero, por favor, notifíqueselo a la autora. La contraseña es "EXP".

${ }^{4}$ Nótese que las tablas correspondientes para el cálculo manual del número de oferentes y demandantes de la versión española del manual para el profesor de Bergstrom y Miller (2008: p. 13) contiene una errata. En la columna "E" de la tabla Q.1.3. el número de "demandantes de elevado valor" correcto es " $2 \mathrm{~N}+2$ ".
} 


\begin{tabular}{|c|c|c|}
\hline \multicolumn{3}{|c|}{ TABLA 1 } \\
\hline TIPO DE JUGADORES (A-F) & SESIÓN 1 & SESIÓN 2 \\
\hline Oferentes de Coste Bajo (CP=10) & 8 & 4 \\
\hline Oferentes de Coste Alto (CP=30) & 4 & 8 \\
\hline Demandantes de Valor Alto (VC=40) & 4 & 8 \\
\hline Demandantes de Valor Bajo & 8 & 4 \\
\hline \multicolumn{2}{|c|}{$N^{0}$ de paquetes completos de 6 (A-F) } & 4 \\
\hline \multicolumn{2}{|c|}{ Tipo de la última hoja de información personal distribuida $=$} & $\mathrm{E}$ \\
\hline
\end{tabular}

Figura 1. Hoja <Tipos> del fichero Excel <exp_naranjas.xls>. Resultados de un experimento.
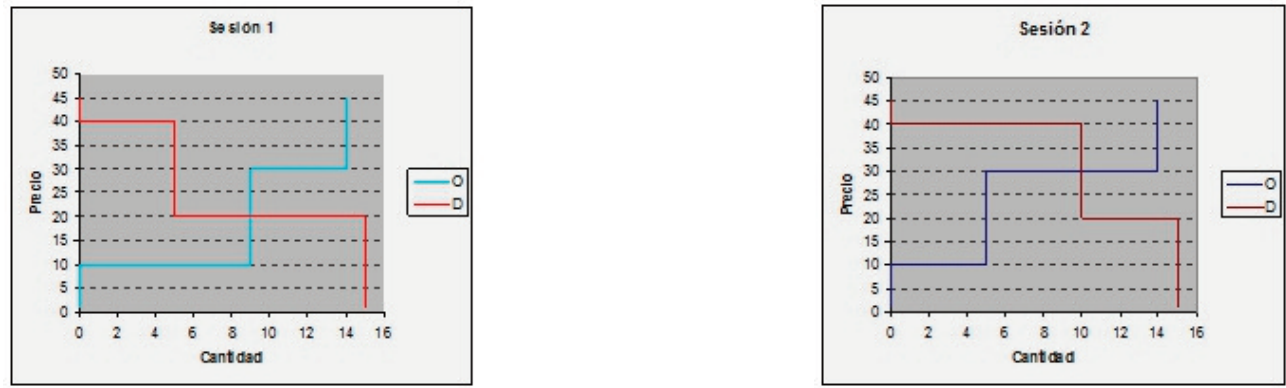

Figura 2. Curvas de oferta y de demanda. Hojas $<$ Tablas 0 y $D>$ y $<$ Teoría vs Evidencia $>$ del fichero Excel <exp_naranjas.xls $>$. Resultados de un experimento.

das del mercado en el que acaban de participar. Estas curvas de oferta y de demanda también se generarán de forma automática en las hojas "Tablas O y D" y "Teoría vs Evidencia" (ver Figura 2).

Las seis hojas siguientes ( $" \mathrm{~S}_{\mathrm{i}}, \mathrm{R}_{\mathrm{j}}$ ”, donde $\mathrm{i}=\{1,2\}$ y $\mathrm{j}=\{1,2,3\}$ denotan, respectivamente, el número de la sesión y de la ronda) permiten registrar las transacciones realizadas en cada experimento sin necesidad de recurrir a los tediosos contratos de compra-venta. Para evitar posibles errores en la introducción de los datos y agilizar al máximo el registro, estas hojas requieren que se introduzcan exclusivamente (i) el precio al que se realiza la transacción, (ii) el número de identificación del vendedor y (iii) el número de identificación del comprador. Las hojas Excel reportan automáticamente el tipo (A-F) de cada jugador registrado de forma que el profesor-notario pueda comprobar rápidamente si los datos que le dictan los jugadores son correctos. Asimismo, detectan el error más habitual, a saber, la confusión entre vendedores y compradores, reportando "FALSO" en tal caso. Siempre de forma automática se calcula el número de transacciones realizadas, el precio medio de las mismas, qué tipo de jugador las ha realizado y qué beneficio (excedente) ha obtenido.
Esta información se traslada de forma automática a la hoja "Teoría vs Evidencia" que permite confrontar las predicciones teóricas del modelo con los resultados experimentales. Por último, la hoja "Resultados" recoge siempre de forma automática los beneficios (excedentes) obtenidos por cada jugador en cada una de las rondas jugadas y calcula su beneficio total y relativo respecto al beneficio que habría podido obtener dado su tipo si todas las transacciones se hubieran realizado a los precios de equilibrio (véase el apéndice).

Finalmente, fue preciso diseñar un sistema de evaluación adecuado. El sistema propuesto consta de dos partes. Con la primera se pretende incentivar la participación responsable de los alumnos. Un adecuado diseño de los incentivos, además de resultar imprescindible en los trabajos de investigación sobre Economía Experimental, mejora los usos docentes de los experimentos puesto que se produce una mayor correspondencia entre los resultados experimentales y las predicciones teóricas (Dickie 2006; Bergstrom y Miller 2008). No obstante, un sistema de incentivos demasiado severo resultaría arbitrario, lo que podría perjudicar el aprendizaje de los alumnos y su evaluación del profeso-

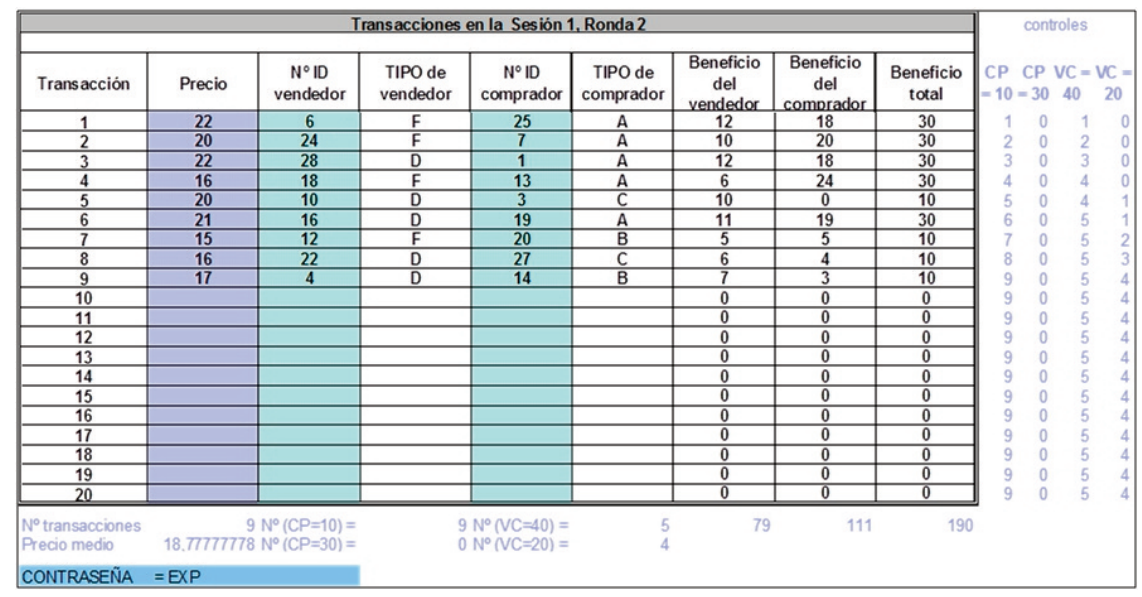

Figura 3. Hoja “S1,R2" del fichero Excel <exp_naranjas.xls>. Resultados de un experimento. 


\begin{tabular}{|l|c|c|c|c|}
\hline SESION 1 & Predicción & Ronda 1 & Ronda 2 & Ronda 3 \\
\hline \hline Precio Medio & 20 & 22,64 & 18,78 & 18,83 \\
\hline $\mathbf{N}^{\circ}$ transaciones & 9 & 11 & 9 & 9 \\
\hline $\mathbf{N}^{0}$ trans aciones vendedores de Coste Bajo (CP=10) & 9 & 9 & 9 & 9 \\
\hline $\mathbf{N}^{0}$ transaciones vendedores de Coste Alto (CP=30) & 0 & 2 & 0 & 0 \\
\hline $\mathbf{N}^{0}$ transaciones de compradores de Valor Alto (VC=40) & 5 & 5 & 5 & 5 \\
\hline $\mathbf{N}^{\mathbf{0}}$ transaciones de compradores de Valor Bajo (VC=20) & 4 & 6 & 4 & 4 \\
\hline Excedente (beneficios) de los vendedores & 90 & 99 & 79 & 79,5 \\
\hline Excedente (beneficios) de los compradores & 100 & 71 & 111 & 110,5 \\
\hline Excedente (beneficios) Total & 190 & 170 & 190 & 190 \\
\hline Eficiencia del mercado experimental & $100 \%$ & $89 \%$ & $100 \%$ & $100 \%$ \\
\hline
\end{tabular}

Figura 4. Hoja “Teoría vs Evidencia” del fichero Excel <exp_naranjas.xls>. Resultados de un experimento.

rado (Dickie 2006). Es por ello que, siguiendo la literatura sobre el tema (véase Fatas y Solaz 2010 para un excelente resumen), se propone incentivar a los alumnos concediéndoles hasta un punto extra en su nota de evaluación continua en función del beneficio total que hayan obtenido en el experimento en relación al que habrían podido obtener en equilibrio dado su tipo (A-F), tal y como se recoge en la tabla del apéndice. Este sistema de evaluación tiene las siguientes ventajas: (i) al conceder puntos extras, palia las injusticias asociadas a un sistema que premia la obtención de beneficios comerciales, cuando estos dependen más de la suerte y las habilidades de negociación de cada jugador que de su conocimiento sobre la materia; (ii) al establecer un sistema de pagos relativo confiriere las mismas oportunidades de ganancia (nota) a todos los jugadores independientemente del cuál sea su tipo (A-F); (iii) al asignar las notas de forma independiente a cada jugador, evita la formación de coaliciones que desvirtuarían el mercado bajo competencia perfecta. ${ }^{5}$ Con la segunda parte de la evaluación se pretende valorar de forma objetiva los conocimientos adquiridos por los estudiantes. Para ello se corregirán los ejercicios sobre los que los alumnos deberán haber trabajado en casa y se les asignará la nota que el profesor considere oportuno (en el caso de la experiencia aquí descrita, la máxima puntuación asignada a la evaluación continua de la sesión en cuestión).

\section{La ejecución y coordinación de la actividad do- cente con carácter de buena práctica}

Como se indica en la sección precedente, este trabajo ofrece un protocolo de uso (protocolo.doc) que detalla paso a paso qué tiene que hacer el profesor en cada momento. A las recomendaciones que allí se especifican podrían añadirse las que, acertadamente, listan Llinares Císcar y Nebot Monferrer (2010) para la mejor implementación del experimento sobre selección adversa propuesto por Bergstrom y Miller (2008). Conviene limitar el número de rondas, utilizar grupos relativamente pequeños de no más de 60 alumnos, disponer de tiempo suficiente para realizar la totalidad de la actividad en una clase de aproximadamente dos horas y apoyarse en un profesor-notario que registre las transacciones. Adicionalmente, se propone realizar el experimento antes de presentar la teoría puesto que el aprendizaje inductivo parece ser más efectivo y, en cualquier caso, la curiosidad natural de los estudiantes por el experimento ayudará a captar su atención durante la explicación en clase. Igualmente importante es mantener el carácter lúdico del experimento puesto que, si bien los experimentos docentes suponen mucho más que juego y diversión (Durham et al. 2007), el entusiasmo que provocan en los estudiantes parece repercutir positivamente sobre las horas de estudio individual y en grupo que le dedican a la materia (Ball et al. 2006: 445). La mayor dificultad de realizar esta actividad docente consiste precisamente en equilibrar la agitación que el juego provoca en los estudiantes con la concentración que requiere la discusión de los resultados experimentales a la luz de la teoría económica. Mantener la disciplina de los alumnos resulta crucial para alcanzar este equilibrio.

Tras probar la idoneidad de la metodología adoptada en un curso experimental, se extendió a todos los cursos de iniciación a la Economía impartidos en la Universidad CEUCardenal Herrera. Ello ha permitido la incorporación del aprendizaje experimental al curriculum de los grados en Dirección de Empresa, Finanzas y Contabilidad, Marketing, Derecho, Ciencias Políticas, Periodismo, Comunicación Audiovisual y Publicidad y Relaciones Públicas. Posteriormente, se realizó la misma actividad docente en grupos más numerosos con alumnos de Economía en la Universidad de Valencia.

\section{Resultados y Conclusiones}

La actividad que se describe en este trabajo ha facilitado la adecuación de los programas y prácticas docentes de la Universidad CEU-Cardenal Herrera y de la Universidad de Valencia al Espacio Europeo de Educación Superior, ha mejorado el proceso de enseñanza-aprendizaje y ha permitido la extensión del uso combinado de experimentos y lecciones a otros profesores con carácter de buena práctica. Una primera valoración de la actividad docente sugiere que, al fomentar el aprendizaje activo e involucrar a los alumnos en el estudio de la materia a través del juego, se ha conseguido que los alumnos desarrollen su capacidad de análisis crítico, discutan los resultados experimentales con sus compañeros, mejorando así su capacidad de comunicación y sus relaciones interpersonales, y consigan mejores resultados académicos.

Desde un punto de vista más específico, todos los profesores involucrados coinciden en que esta actividad ha ayudado a que los alumnos comprendan mejor cómo funciona un mercado perfectamente competitivo y qué supuestos deben darse para que un mercado lo sea. Aparte de introducir una serie de conceptos y métodos básicos para el análisis económico, esta actividad permite que los alumnos observen el papel que los precios juegan como mecanismo descentralizado de asignación y experimenten la dinámica que conduce al equilibrio en un mercado, aspectos ambos dos que difícilmente podríamos explicar con los modelo estáticos de los que se dispone en un curso de iniciación a la Economía. Asimismo, esta actividad permite iniciar a los alumnos en tareas de investigación, enseñándoles a someter a juicio la validez de un modelo confrontando sus supuestos y predic-

${ }^{5}$ En otros contextos, algunos autores han notado los riesgos de introducir un incentivo que premie el rendimiento en función de los logros de los demás jugadores en tanto cuanto esto podría oscurecer algunos puntos clave, como las ganancias mutuas derivadas del comercio, y trivializar algunas formas de comportamiento estratégico y/o ético en juegos cooperativos (Dickie 2006: 268). 
ciones teóricas con la evidencia (experimental) disponible.

Por todo ello, se considera que la implementación de esta actividad docente ha sido un éxito y, como coordinadora del área de Economía de la Universidad CEU-Cardenal Herrera, se propondrá continuar realizándola en el futuro. Para mejor evaluar sus efectos sobre los rendimientos académicos de los estudiantes, será necesario, sin embargo, realizar un estudio controlado que detecte diferencias significativas entre las notas obtenidas y las evaluaciones docentes realizadas por los estudiantes que experimentan con los principios económicos y los que forman parte de un grupo de control.

\section{Bibliografía}

Ball, Sheryl B.; Eckel, Catherine; Rojas, Christian (2006). Technology Improves Learning in Large Principles of Economics Classes: Using Our WITS. American Economic Review, $P \& P$, 96.2, pp. 442-446.

Bergstrom, Theodore C.; Miller, John H. (2008). Experimentos con los principios económicos. Barcelona: Antoni Bosch, 2000.

Dickie, Mark (2006). Do Classroom Experiments Increase Learning in Introductory Microeconomics? Journal of Economic Education, 37, pp. 267-288. http://dx.doi.org/10.3200/JECE.37.3.267-288

Durham, Yvonne; MacKinnon, Thomas; Schulman, Craig (2007). Classroom Experiments: Not Just Fun and Games. Journal of Economic Inquiry, 45.1, pp. 162178. http://dx.doi.org/10.1111/j.14657295.2006.00003.x

Emerson, Tisha. L.; Taylor, Beck A. (2004). Comparing Student Achievement across Experimental and Lecture-Oriented Sections of a Principles of Microeconomics Course. Southern Economic Journal, 70, pp. 672-693. http://dx.doi.org/10.2307/4135338

Fatas, Enrique; Solaz, Héctor (2010). Aprendiendo a jugar Nash con experimentos repetidos. II Jornada de docencia en Economía. Valencia, 28 de abril de 2010 - 2 de mayo de 2010.

González de Lara, Yadira; Pastor Gosálbez, Maite (2010). Material de apoyo para realizar experimentos en clase. Libro de Abstracts de las II Jornadas de Docencia en Economía, 2010. ISBN 978-84-15026-06-8. Recurso electrónico. Editores Teresa García Muñoz, Penélope Hernández Rojas y Ramón María-Dolores.

Llinares Císcar, Juan Vicente; Nebot Montferrer, César (2010). Implementación de un experimento de selección adversa en clase de microeconomía. @tic: revista d'innovació educativa. (4) [Docentic]. http://ojs.uv.es/index.php/attic/article/view/155/199. Fecha de consulta 17/03/2011. 
Apéndice. Hoja "Resultados" del fichero <exp_naranjas.xls>. Resultados de un experimento.

\begin{tabular}{|c|c|c|c|c|c|c|c|c|c|c|}
\hline \begin{tabular}{c|} 
TIPO \\
GANGDOR \\
\end{tabular} & \begin{tabular}{|l|l|l|}
$N$ & $T$ \\
\end{tabular} & Tipo & \begin{tabular}{|c|} 
Beneficio \\
S.1-R.1.
\end{tabular} & $\begin{array}{l}\text { Beneficio } \\
\text { S.1.R2.2. }\end{array}$ & $\begin{array}{l}\text { Beneficio } \\
\text { S.1-R.3. }\end{array}$ & $\begin{array}{l}\text { Beneficio } \\
\text { S.2.R.1. }\end{array}$ & $\begin{array}{l}\text { Beneficio } \\
\text { S.2-R2. }\end{array}$ & $\begin{array}{l}\text { Beneficio } \\
\$ 2 . R 3 .\end{array}$ & $\begin{array}{l}\text { Beneficio } \\
\text { Idtal }\end{array}$ & $\begin{array}{l}\text { Beneficio } \\
\text { Relativo }\end{array}$ \\
\hline IPh & & & 20 & 品 & 勿 & ס & ס & & - & \\
\hline & 1 & $\mathbf{A}$ & D & 18 & 2 & D & D & & 3 & EST \\
\hline & 7 & A & 11 & (2) & 18 & 1 & DE & & 505 & 8.5 \\
\hline & 13 & A & 21 & 24 & בan & D & & & 亩 & $11 \mathrm{iz}$ \\
\hline & 19 & & D & 19 & 21 & D & & & 5 & $\sqrt{5}$ \\
\hline & 2 & $A$ & D & 18 & 2 & 1 & $\underline{D}$ & & 3 & $\frac{105}{5-2}$ \\
\hline & 3 & $\mathbf{A}$ & 9 & 0 & D & $\overline{\mathbf{D}}$ & D & & 9 & $\frac{\pi}{\sqrt{7}}$ \\
\hline & 37 & & D & 0 & D & $\overline{\mathbf{D}}$ & $\bar{D}$ & & $\mathbf{D}$ & $\frac{57}{157}$ \\
\hline & 5 & $\mathbf{A}$ & D & $\mathbf{0}$ & $\mathbf{0}$ & $\mathbf{D}$ & $\frac{D}{0}$ & & $\overline{0}$ & $\frac{0 z}{7}$ \\
\hline & 2 & A & 7 & D & D & $\frac{0}{n}$ & 0 & & $\frac{8}{n}$ & $\frac{\pi}{\pi 7}$ \\
\hline & (3) & $\boldsymbol{n}$ & & & & & & & & \\
\hline TPBWE=2_CP-1M & & & D & 可 & 0 & 2 & 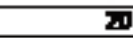 & & 0 & 100 \\
\hline & 2 & 旦 & D & D & D & 15 & 15 & & 30 & $\overline{\bar{n}}$ \\
\hline & 8 & 日 & E & D & D & 125 & 175 & & 0 & 1007 \\
\hline & 14 & 奇 & D & 3 & 4 & 15 & 18.5 & & 05 & 1017 \\
\hline & an & & D & & 3 & $\frac{13}{14}$ & D & & 21 & $\frac{597}{77}$ \\
\hline & $\mid$ & 早 & D & 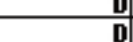 & $\frac{\mathbf{D}}{\mathrm{D}}$ & $\frac{11}{\mathrm{D}}$ & $\frac{16}{0}$ & & $\frac{31}{0}$ & $\frac{\pi}{07}$ \\
\hline & 衤 & 量 & D & 0 & D & D & D & & D & $\frac{D z}{0 z}$ \\
\hline & 4 & E & D & D & D & D & D & & D & \\
\hline & [50: & 旦 & D] & D] & D] & D & D & & D & \\
\hline & 困 & B & D & D & D & D & D & & D & 07 \\
\hline 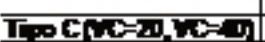 & & & D & 可 & J & 10 & 10 & & 7 & 10,0 \\
\hline & \begin{tabular}{|l|}
3 \\
\end{tabular} & $\mathrm{C}$ & 2 & D & D & $\frac{15}{9}$ & 15 & & 3 & 10 \\
\hline & \begin{tabular}{|c|} 
\\
\end{tabular} & $\mathrm{C}$ & D & D & D & 9 & 95 & & 185 & 57 \\
\hline & 15 & C & 5 & D & 2 & $\frac{10}{10}$ & 115 & & 285 & 167 \\
\hline & 21 & $c$ & 5 & 4 & $\frac{2,5}{0}$ & $\frac{13,5}{10}$ & $\frac{12,5}{15}$ & & $\frac{33,5}{7}$ & $168 \%$ \\
\hline & $\mid \frac{25}{33}$ & $\mathrm{c}$ & D & D & $\mathbf{D}$ & D & D & & a & $\frac{12 \sqrt{2}}{0 z}$ \\
\hline & 3i & C & D. & D & D & D & D & & D & 05 \\
\hline & -6 & $\mathbf{C}$ & D & D & D & D & D & & D & $0 \mathbf{z}$ \\
\hline & 범 & c & D. & D & D] & D & D & & D & 05 \\
\hline & Fi & C & D & D & D & D & D & & D & 05 \\
\hline 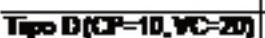 & & & 10 & 10 & 10 & 0 & 0 & & $\mathbf{3}$ & \\
\hline & a & D & $\mathbf{z}$ & 7 & 10 & D & D & & $\overline{0}$ & 1337 \\
\hline & 10 & D & 9 & 10 & 7.5 & D & D & & 25 & 87 \\
\hline & 18 & D & 19. & 11 & 12 & D & D & & 8 & 107 \\
\hline & $\mathbf{z}$ & D & 5 & 目 & 10 & D & D & & 2 & $\pi 78$ \\
\hline & 28 & $\mathbf{D}$ & D & 12 & 8 & D & D & & 21) & \\
\hline & 34 & $\mathbf{D}$ & D. & D. & D & $\underline{\mathbf{D}}$ & D & & $\overline{\mathbf{D}}$ & $\overline{\mathbf{D z}}$ \\
\hline & - & D & D] & D] & D] & D & D & & D & \\
\hline & is & D & D & D] & D] & D & D & & D & \\
\hline & $5 \mathbf{2}$ & D & D. & D & D] & D & D & & D & 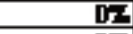 \\
\hline & 四 & D & D & D] & D] & D & D & & D & $0 \mathbf{z}$ \\
\hline TSE & & & D & 可 & 可 & 10 & 10 & & $\overline{0}$ & $10 \mathrm{~W}$ \\
\hline & 5 & $\mathbf{E}$ & (1) & $\mathbf{D}$ & D & 10 & 95 & & 195 & \\
\hline & 11 & $E$ & D. & D & D & 19 & $\mathbf{9}$ & & $\underline{\mathbf{z}}$ & $19 \sqrt{7}$ \\
\hline & 17] & $E$ & 10 & D] & D] & 15 & 5 & & 3) & 1:Firz \\
\hline & $\mathbf{z}$ & $E$ & 1 & D & D & 9 & 10 & & $\underline{\mathbf{2}}$ & 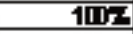 \\
\hline & $\mathbf{Z}$ & $\mathbf{E}$ & D & D & D & 17 & 10 & & $\underline{\mathbb{Z}}$ & 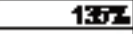 \\
\hline & 3: & E & D & D & D & D & D & & D & 07 \\
\hline & +1 & $\mathrm{E}$ & D & D & D & D & D & & D & Dr \\
\hline & 7 & $E$ & D & D] & D] & D & D & & D & $D \mathbf{z}$ \\
\hline & [5] & $E$ & D & D & D] & D & D & & D & Dr \\
\hline & 因 & E & D. & D & D & D & D & & D & $0 \mathbf{z}$ \\
\hline 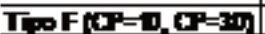 & $t$ & & 10 & 10 & 10 & $\overline{0}$ & 0 & & $\overline{3}$ & 100. \\
\hline & & $F$ & & 12 & & D & D & & $\overline{\mathbf{z}}$ & \\
\hline & 12: & $F$ & 10 & 5 & 10 & $\overline{\mathbf{D}}$ & 1 & & 至 & $\frac{6}{8 x}$ \\
\hline & 18 & $F$ & 4 & 百 & 7 & $\overline{\mathbf{D}}$ & 5 & & $\overline{\mathbf{z}}$ & $\overline{B_{7}}$ \\
\hline & 24 & $F$ & 5 & 10 & 9 & $\overline{\mathbf{D}}$ & D5 & & 245 & 87 \\
\hline & 30 & $\mathbf{F}$ & D & D & D & D & D & & D & 07 \\
\hline & [3] & $\mathbf{F}$ & D] & D] & D] & D & D & & D & Drz \\
\hline & 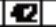 & $F$ & D. & D] & D] & D & D & & D & $0 \mathbf{z}$ \\
\hline & 8 & $F$ & D & D & D & $\mathbf{D}$ & D & & D & \\
\hline & 59 & $\mathbf{F}$ & D & D & $\mathbf{D}$ & $\frac{D}{n}$ & D & & D & $\frac{05}{27}$ \\
\hline & 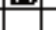 & 1 & & & & & & & & 焉 \\
\hline
\end{tabular}

